\title{
Collective oscillations of globally coupled bistable, nonresonant components
}

\author{
Munir Salman $\odot,{ }^{1}$ Christian Bick, ${ }^{2}$ and Katharina Krischer $\circledast^{1, *}$ \\ ${ }^{1}$ Physik-Department, Technische Universität München, James-Franck-Straße 1, 85748 Garching, Germany \\ ${ }^{2}$ Centre for Systems Dynamics and Control and Department of Mathematics, University of Exeter, Exeter EX4 4QF, United Kingdom; \\ Institute for Advanced Study, Technische Universität München, Lichtenbergstraße 2, 85748 Garching, Germany; \\ and Oxford Centre for Industrial and Applied Mathematics, Mathematical Institute, University of Oxford, Oxford OX2 6GG, United Kingdom
}

(Received 10 January 2020; accepted 30 September 2020; published 23 October 2020)

\begin{abstract}
Bistable microelectrodes with an S-shaped current-voltage characteristic have recently been shown to oscillate under current control, when connected in parallel. In other systems with equivalently coupled bistable components, such oscillatory instabilities have not been reported. In this paper, we derive a general criterion for when an ensemble of coupled bistable components may become oscillatorily unstable. Using a general model, we perform a stability analysis of the ensemble equilibria, in which the components always group in three or fewer clusters. Based thereon, we give a necessary condition for the occurrence of collective oscillations. Moreover, we demonstrate that stable oscillations may persist for an arbitrarily large number of components, even though, as we show, any equilibrium with two or more components on the middle, autocatalytic branch is unstable.
\end{abstract}

DOI: 10.1103/PhysRevResearch.2.043125

\section{INTRODUCTION}

In contrast to ensembles of coupled oscillators, which have been extensively studied for decades, we have just started to recognize the variety of responses coupled bistable components can exhibit. Kouvaris and colleagues investigated tree networks of bistable nodes where the individual nodes, or bistable components, were diffusively coupled [1-3]. They demonstrated that such a network can form a self-organized stationary pattern, similar to a Turing pattern. Another peculiar behavior, reported for an array of bistable electrodes [4,5] as well as for nanoparticulate Li-ion batteries [6-10], is that a slow parameter ramp leads to a sequential one-by-one passage of an individual element from one state-or phase-to the other one. Surprisingly, oscillations were also observed in such all-to-all connected ensembles of a few bistable components at fixed parameter values [11]. This finding was the motivation of the present study, where we derive a necessary condition for the occurrence of a Hopf bifurcaton in systems composed of all-to-all connected generic bistable components and demonstrate that oscillatory dynamics may also persist in the macroscopic limit.

Before introducing our general mathematical formulation, it is helpful to elucidate the physical setting with a particular system. We consider $\mathrm{CO}$ electro-oxidation on an array of $\mathrm{Pt}$ electrodes (or many Pt nanoparticles on a conducting support) which are all controlled through a common external circuit.

\footnotetext{
*Corresponding author: krischer@tum.de

Published by the American Physical Society under the terms of the Creative Commons Attribution 4.0 International license. Further distribution of this work must maintain attribution to the author(s) and the published article's title, journal citation, and DOI.
}

First consider the dynamics of an individual electrode (or nanoparticle) [12]. The reaction kinetics of $\mathrm{CO}$ oxidation on $\mathrm{Pt}$ is autocatalytic, promoting the coexistence of a state with a high $\mathrm{CO}$ coverage and a state with a low $\mathrm{CO}$ coverage at a given set of parameters. The overall reaction rate, and thus the current, is high if the CO coverage is low, and vice versa. Hence each individual electrode, or nanoparticle, also exhibits bistable steady-state current-voltage characteristics, as indicated in Fig. 1(a). When slowly increasing the external voltage from an initially low value, the system eventually jumps from the low-current state to the high-current state. Upon reversing the scan direction, the transition back to the low-current state occurs at a lower value of the external voltage. As a result, the steady-state current-potential curve exhibits a hysteresis, but the system always approaches a spatially uniform stationary state. The situation becomes fundamentally different when instead of the external voltage, the total current is controlled [13]. When the set current value corresponds to an intermediate value between the two stable branches, the $\mathrm{CO}$ coverage splits into two regions, one where the electrode attains a high-CO-coverage-low-current state and the other where it attains a low-CO-coverage-high-current state, and the voltage adjusts to a value within the bistable regime at which both states are equally stable. A change of the set current changes the ratio of high-coverage to low-coverage domains, while the voltage stays at the same value, until one of the two domains extends over the entire electrode. This behavior can be seen as a nonequilibrium analog to first-order phase transitions. However, for the domain formation to occur, the electrode area has to exceed a critical size. Individual, sufficiently small electrodes trace out the S-shaped current-voltage equilibrium under current control [14]. This is the behavior we consider for an individual, bistable component in our general model.

Now consider many such electrodes connected in parallel to the same external electric circuit under galvanostatic 
(a)
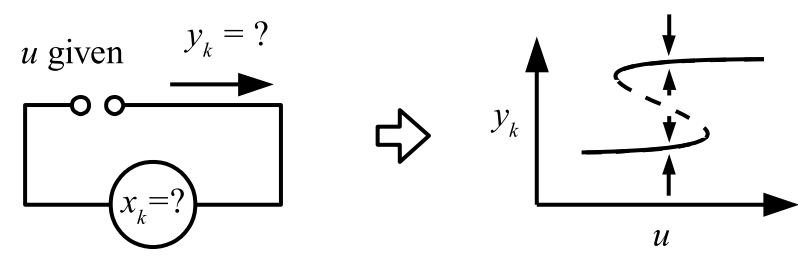

(b)
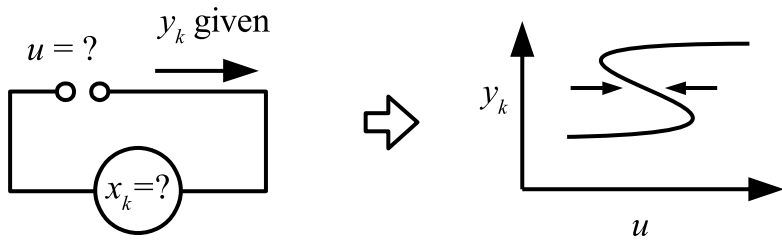

(c)

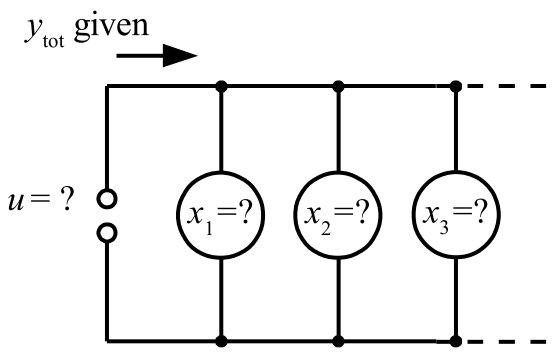

FIG. 1. Circuit representation and $y_{k}-u$ curve of an individual bistable component (a) under $u$ control and (b) under $y_{k}$ control. (c) Circuit representation of many bistable components under $y_{\text {tot }}$ control.

control; that is, the total current through all electrodes is controlled, but the partial currents through the individual electrodes may differ from each other. This is the situation we refer to as an all-to-all coupled bistable multicomponent ensemble. Starting at a low value of the total current where all the partial currents attain the same low value, a slow current ramp induces one electrode after the other to undergo a transition from the low- to the high-current state. These sequential transitions might now occur in two different ways: In the simpler scenario, each individual electrode resumes one of the three steady states of the S-shaped current-potential curve, the voltage adopting correspondingly to a fixed value within the hysteretic region [5]. In the other scenario, the individual electrodes also split into two or three clusters, but the voltage as well as the partial currents undergo stable oscillations [11]. The oscillations were observed for a small number of electrodes and could be reproduced with a specific model. The main result of our paper is new insight into how oscillations in bistable systems occur. Specifically, we outline the necessary properties of the kinetics of a bistable system in order to support oscillations and demonstrate that oscillations may persist in systems composed of a macroscopically large number of components. Our results elucidate whether important technologically relevant systems, e.g., in catalysis or electrocatalysis where the catalytically active ma- terial consists of nanoparticles that might have a bistable reaction kinetics [15], can exhibit oscillations.

The remainder of this paper is organized as follows. In the next three sections, we consider a generic bistable model. After defining our mathematical system in general terms in the next section, we present a stability analysis of its stationary states, and, in particular, we present a proof that in a stable hyperbolic stationary state there is at most one element on the autocatalytic branch. Then, we derive a general, necessary condition for the occurrence of a Hopf bifurcation in this type of system and illustrate this condition with an intuitive $L C$ circuit analog. Thereafter, we validate the predictions with simulations of a model system, derived from a more complex model for $\mathrm{CO}$ electro-oxidation. We demonstrate the emergence of oscillations from a Hopf bifurcation in a small system. Moreover, we illustrate that in arbitrary large systems an unstable limit cycle can be stabilized.

\section{EMERGENCE OF OSCILLATIONS IN SYSTEMS OF BISTABLE ELEMENTS}

\section{A. Model equations}

First, consider the mathematical formulation of an individual component subject to control through a parameter $u \in \mathbb{R}$.

We represent the $k$ th bistable component of our ensemble in the following state space form, in which $x_{k} \in \mathbb{R}$ is the inner state of the system, $u \in \mathbb{R}$ is the control parameter, and a variable $y_{k} \in \mathbb{R}$ adjusts accordingly [cf. Fig. 1(a)]. Our analysis was inspired by an electrochemical setup, so we call $u$ voltage and $y_{k}$ current, but the upcoming results and circuit representations are valid for other physical realms as well. In the electrochemical context, $x_{k}$ can be thought of as the surface fraction of the $k$ th electrode that is covered with a certain chemical species.

$$
\begin{gathered}
\frac{d}{d t} x_{k}(t)=f\left(x_{k}(t), u\right), \\
y_{k}(t)=g\left(x_{k}(t), u\right) .
\end{gathered}
$$

The function $f$ is a bistable flow field of $x_{k}$. The current $y_{k}$ is an observable depending on the inner state $x_{k}$ and $u$. The equilibrium curve is $\mathrm{S}$ shaped in the $y_{k}-u$ plot.

Next, assume we have an ensemble of $n$ such components, indexed by $k$, all identical. We are ultimately interested in what happens if the total current $y_{\text {tot }}$ is fixed [see Fig. 1(c)], where

$$
y_{\mathrm{tot}}=\sum_{k=1}^{n} y_{k}(t) .
$$

So we end up with

$$
\begin{aligned}
& \frac{d}{d t} x_{k}(t)=f\left(x_{k}(t), u(t)\right), \\
& y_{\text {tot }}=\sum_{k=1}^{n} g\left(x_{k}(t), u(t)\right),
\end{aligned}
$$

where now $u(t)$ adjusts instantaneously such that Eq. (5) is always fulfilled. 


\section{B. Linear stability analysis}

Before we come to the case in which the coupling between the components is obtained by fixing $y_{\text {tot }}$, let us first quickly consider the simpler case in which $u$ is controlled, rather than $y_{\text {tot }}$. If $u$ is controlled, the components are uncoupled and just follow Eqs. (1) and (2), each. Correspondingly, the ensemble equilibria at each value of $u$ are determined by all combinations of how the $n$ components can distribute between the three available component equilibria, which we assumed to exist for Eqs. (1) and (2). We denote the component equilibria by $x^{\mathrm{eq}, 1}, x^{\mathrm{eq}, 2}, x^{\mathrm{eq}, 3}$. Furthermore, let $y_{\text {tot }}^{\mathrm{eq}}$ denote the equilibrium value of $y_{\text {tot }}(t)$, and let $u^{\text {eq }}$ denote the prescribed value of $u$.

Linearizing around the $n$-dimensional ensemble equilibrium and denoting small deviations by a circumflex (also known as a "hat"), we arrive at Eqs. (6) and (7), using the definitions in Eqs. (8)-(13), in which $\left\{\mathbf{e}_{1}, \ldots, \mathbf{e}_{n}\right\}$ is a standard basis, $k \in\{1, \ldots, n\}$ indexes components, and $\ell_{k} \in\{1,2,3\}$ indexes the cluster of the $k$ th component.

$$
\begin{gathered}
\frac{d}{d t} \hat{\mathbf{x}}(t)=F_{x} \hat{\mathbf{x}}(t)+F_{u} \hat{u}, \\
\hat{y}_{\mathrm{tot}}(t)=G_{x} \hat{\mathbf{x}}(t)+G_{u} \hat{u}, \\
\hat{\mathbf{x}}(t)=\sum_{k=1}^{n}\left[x_{k}(t)-x^{\mathrm{eq}, \ell_{k}}\right] \mathbf{e}_{k} \in \mathbb{R}^{n}, \\
\hat{u}=u-u^{\mathrm{eq}}, \quad \hat{y}_{\mathrm{tot}}(t)=y_{\mathrm{tot}}(t)-y_{\mathrm{tot}}^{\mathrm{eq}}, \\
F_{x}=\operatorname{diag}\left(f_{x}\left(x^{\mathrm{eq}, \ell_{1}}\right), \ldots, f_{x}\left(x^{\mathrm{eq}, \ell_{n}}\right)\right) \in \mathbb{R}^{n \times n}, \\
F_{u}=\sum_{k=1}^{n} f_{u}\left(x^{\mathrm{eq}, \ell_{k}}\right) \mathbf{e}_{k} \in \mathbb{R}^{n \times 1}, \\
G_{x}=\sum_{k=1}^{n} g_{x}\left(x^{\mathrm{eq}, \ell_{k}}\right) \mathbf{e}_{k}^{\mathrm{T}} \in \mathbb{R}^{1 \times n}, \\
G_{u}=\sum_{k=1}^{n} g_{u}\left(x^{\mathrm{eq}, \ell_{k}}\right) \in \mathbb{R}^{1 \times 1} .
\end{gathered}
$$

Here, we used the abbreviations

$$
\begin{aligned}
& f_{x}\left(x^{\mathrm{eq}, \ell}\right)=\partial f /\left.\partial x_{k}\right|_{x^{\mathrm{eq}, \ell}, u^{\mathrm{eq}},} \\
& f_{u}\left(x^{\mathrm{eq}, \ell}\right)=\partial f /\left.\partial u\right|_{x^{\mathrm{eq}, \ell}, u^{\mathrm{eq}},} \\
& g_{x}\left(x^{\mathrm{eq}, \ell}\right)=\partial g /\left.\partial x_{k}\right|_{x^{\mathrm{eq}, \ell}, u^{\mathrm{eq}},}, \\
& g_{u}\left(x^{\mathrm{eq}, \ell}\right)=\partial g /\left.\partial u\right|_{x^{\mathrm{eq}, \ell}, u^{\mathrm{eq}} .} .
\end{aligned}
$$

Hence, under $u$ control, the stability of the ensemble state follows directly from the stability of the individual component equilibria, the ensemble equilibrium being only stable if all individual components are on one of the two outer stable branches of the S-shaped equilibrium curve.

Let us now consider the more interesting case in which the total current $y_{\text {tot }}$, instead of $u$, is prescribed as control parameter [see Fig. 1(c) and Eq. (3)]. In this case, $u$ adjusts such that the constraint on $y_{\text {tot }}$ is fulfilled. The equilibria remain unchanged, but their stability may alter and the dynamics change to

$$
\begin{gathered}
\frac{d}{d t} \hat{\mathbf{x}}(t)=\overbrace{\left(F_{x}-F_{u} G_{u}^{-1} G_{x}\right)}^{=: \tilde{x}_{x}} \hat{\mathbf{x}}(t)+\overbrace{F_{u} G_{u}^{-1}}^{=: \tilde{y}_{\mathrm{tot}}}, \\
\hat{u}(t)=\underbrace{-G_{u}^{-1} G_{x}}_{=: \tilde{G}_{x}} \hat{\mathbf{x}}(t)+\underbrace{G_{u}^{-1}}_{=: \tilde{G}_{y}} \hat{y}_{\mathrm{tot}} .
\end{gathered}
$$

This is derived by solving Eq. (7) for $\hat{u}$ and eliminating $\hat{u}$ in Eq. (6). Thus we have obtained the Jacobian $\tilde{F}_{x}$ of the $y_{\text {tot }}{ }^{-}$ controlled ensemble. The approach we took in finding it was inspired by well-established control theory: defining a state space equation, linearizing it at a fixed point, and rearranging it to a conjugate version in which $u$ and $y_{\text {tot }}$ are interchanged [16].

What can we infer about the stability of an ensemble equilibrium under $y_{\text {tot }}$ control, when only the properties of the individual components are given? This is asking how the eigenvalues of the Jacobian $\widetilde{F}_{x}$ depend on the derivatives $f_{x}, f_{u}, g_{x}, g_{u}$. Whereas under $u$ control [Eqs. (6) and (7)], the stability was determined by a diagonal Jacobian $F_{x}$, now we have $\tilde{F}_{x}$, which has two types of eigenvectors, as in the case of mean-field-coupled identical Stuart-Landau oscillators [17]: the intracluster eigenvectors and the intercluster eigenvectors (see Appendix A for a detailed derivation). The intracluster eigenvectors affect only components at one of the three equilibria $x^{\mathrm{eq}, 1}, x^{\mathrm{eq}, 2}, x^{\mathrm{eq}, 3}$ at a time. Perturbations in their directions only affect elements in a single cluster. By contrast, perturbations in the direction of the intercluster eigenvectors affect at least two clusters but move each cluster as a whole, i.e., they conserve the clusters. We indicate intracluster eigenvectors and eigenvalues by a " $\perp$," as they are perpendicular to the cluster-conserving manifold. Analogously, intercluster eigenvectors and eigenvalues get a " $\|$."

Intracluster eigenvectors $\mathbf{v}_{\ell}^{\perp}$ of $\tilde{F}_{x}$ each have nonzero entries at only one of the three equilibria $x^{\mathrm{eq}, \ell}$, and the entries sum up to zero, i.e., they do not change a cluster's "center of mass." The respective intracluster eigenvalues $\lambda_{\ell}^{\perp}$ of $\tilde{F}_{x}$ have a multiplicity of $\max \left(n_{\ell}-1,0\right)$, in which $n_{\ell}$ is the number of components in the respective cluster $\ell$. Furthermore, the $\lambda_{\ell}^{\perp}$ turn out to match the eigenvalues of the $u$-controlled system, which are identical to those of the $u$-controlled, single component (see Appendix A):

$$
\begin{gathered}
\left(\tilde{F}_{x}-\lambda_{\ell}^{\perp} \mathbf{1}\right) \mathbf{v}_{\ell}^{\perp}=0, \\
\lambda_{\ell}^{\perp}=f_{x}\left(x^{\mathrm{eq}, \ell}\right) .
\end{gathered}
$$

This result has a very important implication: Any ensemble equilibrium under $y_{\text {tot }}$ control is unstable if it has more than one component at a component equilibrium $x^{\text {eq }, \ell}$ that is unstable for a $u$-controlled, single component, i.e., if $n_{\ell}>1$ and $f_{x}\left(x^{\mathrm{eq}, \ell}\right)>0$. This statement generalizes the "mosaic instability" [18] to far-from-equilibrium systems, and it is the reason that components switch individually when the parameter $y_{\text {tot }}$ is ramped. Note that a perturbation by an intracluster eigenvector $\mathbf{v}_{\ell}^{\perp}$ leaves $u$ constant, unlike a perturbation by an intercluster eigenvector $\mathbf{v}_{\ell}^{\|}$. 
To find the intercluster eigenvalues $\lambda^{\|}$that have an impact on $u$, imagine a perturbation affecting the voltage $u$ while the current $y_{\text {tot }}$ is kept constant. This requires that the system has an infinite impedance $Z_{\text {tot }}$. More specifically, the poles of the impedance $Z_{\text {tot }}(\lambda)$ are the intercluster eigenvalues $\lambda^{\|}$. This follows from the definition of the impedance, which involves the Laplace transformation $\mathcal{L}$ :

$$
\begin{aligned}
Z_{\mathrm{tot}}(\lambda) & =\frac{\mathcal{L}\{\hat{u}\}(\lambda)}{\mathcal{L}\left\{\hat{y}_{\mathrm{tot}}\right\}(\lambda)} \\
& =-\tilde{G}_{x}\left(\tilde{F}_{x}-\lambda \mathbf{1}\right)^{-1} \tilde{F}_{u}+\tilde{G}_{u} .
\end{aligned}
$$

We derived (23) from (18) and (19), using $\mathcal{L}\left\{\frac{d}{d t} \hat{\mathbf{x}}\right\}\left(\lambda^{\|}\right)=$ $\lambda^{\|} \mathcal{L}\{\hat{\mathbf{x}}\}\left(\lambda^{\|}\right)$. The poles of the impedance $Z_{\text {tot }}(\lambda)$ can be found by looking for the roots of its reciprocal, i.e., the admittance $Y_{\text {tot }}(\lambda)$ :

$$
\begin{aligned}
0 & =Y_{\text {tot }}\left(\lambda^{\|}\right)=\frac{\mathcal{L}\left\{\hat{y}_{\text {tot }}\right\}\left(\lambda^{\|}\right)}{\mathcal{L}\{\hat{u}\}\left(\lambda^{\|}\right)} \\
& =-G_{x}\left(F_{x}-\lambda^{\|} \mathbf{1}\right)^{-1} F_{u}+G_{u} \\
& =\sum_{\ell \in\{1,2,3\}} \underbrace{n_{\ell}\left[-\frac{g_{x}\left(x^{\mathrm{eq}, \ell}\right) f_{u}\left(x^{\mathrm{eq}, \ell}\right)}{f_{x}\left(x^{\mathrm{eq}, \ell}\right)-\lambda \|}+g_{u}\left(x^{\mathrm{eq}, \ell}\right)\right]}_{=Y_{\ell}\left(\lambda^{\|}\right)} .
\end{aligned}
$$

If the intercluster eigenvalues $\lambda^{\|}$are known, the corresponding eigenvectors $\mathbf{v}^{\|}$are obtained as

$$
\left(\tilde{F}_{x}-\lambda^{\|} \mathbf{1}\right) \underbrace{\left(F_{x}-\lambda^{\|} \mathbf{1}\right)^{-1} F_{u}}_{=\mathbf{v}^{\|}, \text {eigenvec of } \tilde{F}_{x}}=0,
$$

The number of intercluster eigenvectors matches the number of nonempty clusters; hence there are three at most. All other eigenvectors are intracluster eigenvectors.

\section{Existence of oscillations}

Equation (26) thus can be utilized to find instabilities of the ensemble equilibrium with respect to intercluster perturbations. In particular, it helps to find a transition from a damped to a self-amplifying oscillation, i.e., the occurrence of a Hopf bifurcation, marked by a purely imaginary pair of complexconjugate eigenvalues $\lambda_{\mathrm{H}}^{\|}, \lambda_{\mathrm{H}}^{\| *}$ of the Jacobian $\tilde{F}_{x}$ [19]. The frequency of the resulting oscillation is $\omega /(2 \pi)$ and obeys $i \omega=\lambda_{\mathrm{H}}^{\|}=-\lambda_{\mathrm{H}}^{\| *}$, in which $i$ is the imaginary unit.

If we insert $i \omega$ for $\lambda^{\|}$, each of the three clusters has an admittance $Y_{\ell}(i \omega)=Y_{\ell}(-i \omega)^{*}$. A cluster's admittance $Y_{\ell}(i \omega)$ is just the sum of the admittances of its components, $\frac{1}{n_{\ell}} Y_{\ell}(i \omega)$.

The impedance of each of the clusters can be represented as one of the two circuits in Figs. 3(a) and 3(b), in which the choice between the inductive version [Fig. 3(a), orange] and the capacitive version [Fig. 3(b), blue] depends on the sign of the product $g_{x} f_{u}$, as further explained in Appendix B. Intuitively, one would expect that for an oscillation to occur, one would need an $L C$ circuit, which requires both an inductance and a capacitance. In fact, this intuitive requirement for a Hopf bifurcation is also actually found when looking at the imaginary part of Eq. (26):

$$
0=\sum_{\ell \in\{1,2,3\}} \underbrace{-n_{\ell} g_{x}\left(x^{\mathrm{eq}, \ell}\right) f_{u}\left(x^{\mathrm{eq}, \ell}\right) \frac{i \omega}{\left|f_{x}\left(x^{\mathrm{eq}, \ell}\right)-i \omega\right|^{2}}}_{\operatorname{Im}\left[Y_{\ell}(i \omega)\right]} .
$$

(a)
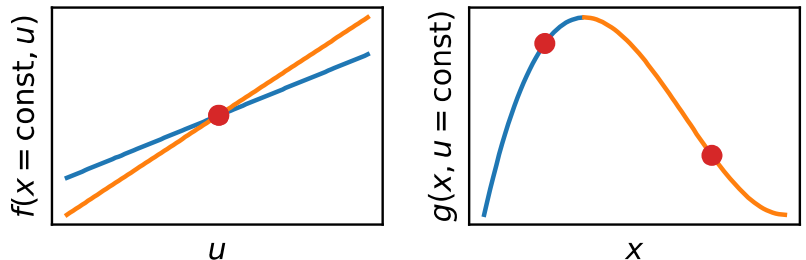

(b)
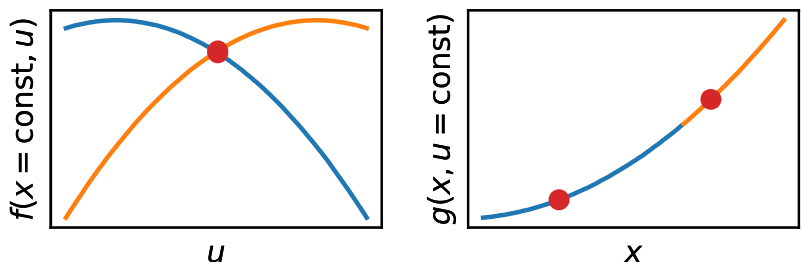

FIG. 2. (a) and (b) Sketch of the two cases in which sustained oscillations can occur with a fixed $y_{\text {tot }}$. A red dot represents a cluster of components.

This equation needs to hold at a Hopf bifurcation. It can only be fulfilled if we have both a cluster $\ell_{1}$ with $g_{x}\left(x^{\mathrm{eq}, \ell_{1}}\right) f_{u}\left(x^{\mathrm{eq}, \ell_{1}}\right)<0$ and a cluster $\ell_{2}$ with $g_{x}\left(x^{\mathrm{eq}, \ell_{2}}\right) f_{u}\left(x^{\mathrm{eq}, \ell_{2}}\right)>0$ such that the total sum is zero. (All summands may also be zero, but this is a degenerate case.)

Thus, the necessary condition for a Hopf bifurcation in the considered class of systems, our core result, is given by

$$
\begin{aligned}
\exists u: \exists x_{1}, x_{2}: & f\left(x_{1}, u\right)=f\left(x_{2}, u\right)=0 \\
& \wedge \partial g /\left.\partial x_{k}\right|_{x_{1}, u} \partial f /\left.\partial u\right|_{x_{1}, u}<0 \\
& \wedge \partial g /\left.\partial x_{k}\right|_{x_{2}, u} \partial f /\left.\partial u\right|_{x_{2}, u}>0 .
\end{aligned}
$$

Condition (29) tells us that in order to find a Hopf bifurcation in a $y_{\text {tot }}$-controlled ensemble of bistable components we need the product $g_{x} f_{u}$ to change its sign along the equilibrium curve of the individual components.

Interestingly, condition (29) depends only on the equations that describe the individual components. It is thus straightforward to test for the possibility of oscillations once a model for the individual component is known. Figure 2 illustrates the two qualitatively different cases which might lead to oscillations. In the first case, $\partial f / \partial u$ has the same sign everywhere along the steady-state curve. Correspondingly, in order to fulfill condition (29), $g(x, u=$ const $)$ has to possess at least one extremum at a given value of $u$ in the bistable regime. In the second case [Fig. 2(b)], $\partial f / \partial u$ is negative on one branch and positive on another. Correspondingly, oscillations might occur also for a strictly monotonic $g(x, u=$ const $)$.

The above-discussed results on the stability of steady states have important implications for the stability of oscillations born in a Hopf bifurcation, which we assume to be supercritical: Stable oscillations can only emerge if the bifurcating steady state has at most one component on the middle, unstable branch of the $y$ - $u$ curve. However, because for a macroscopic number of components the influence of an individual component on the total current is negligible, in systems with a large number of components, a stable limit cycle can only emerge in a Hopf bifurcation if all components of the steady states are in one of the two outer, stable states 
(a)

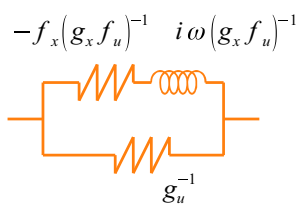

(b)

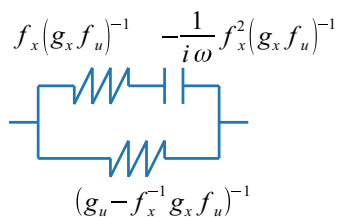

(c)

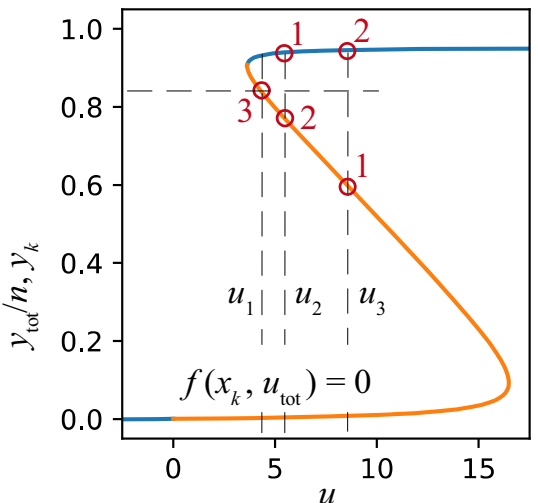

(d)
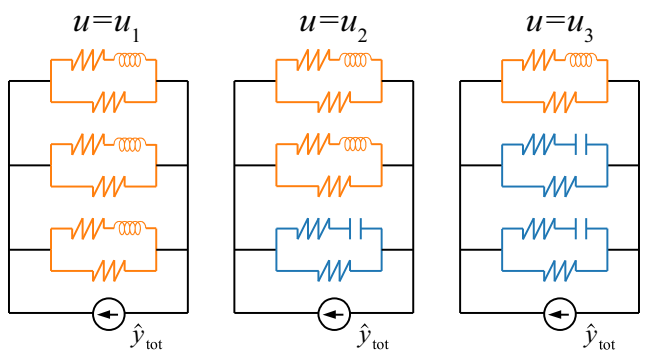

(e)
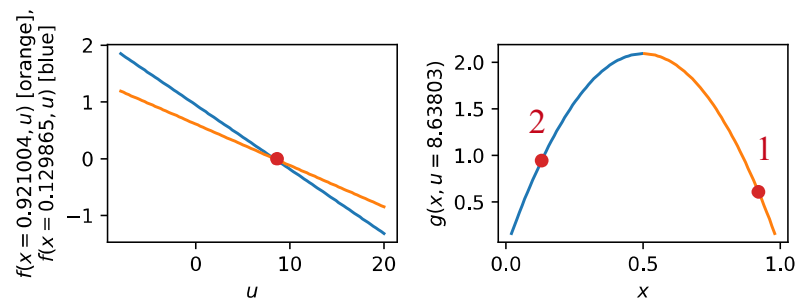

FIG. 3. (a) and (b) Equivalent circuits of a state space model with $x \in \mathbb{R}$, close to equilibrium. (c) Exemplary illustration of the three equilibria of Eqs. (30) and (31) for $n=3, a=0.05, b=0.01$ at $y_{\text {tot }}=0.833 n$, marked by the horizontal dashed line. Red circles indicate clusters of up to three components. (d) Equivalent circuits of the entire ensemble at the equilibria in (c). (e) Function $g$ for $u=u_{3}$ and function $f$ with $x$ fixed to the respective equilibrium values at $u=u_{3}$

of the $y-u$ characteristic. However, below we will demonstrate that initially unstable limit cycles might be stabilized in an equivariant transcritical bifurcation, and therefore stable oscillations also exist for two- and three-cluster states with an arbitrarily large number of components in the middle, unstable state. (a)
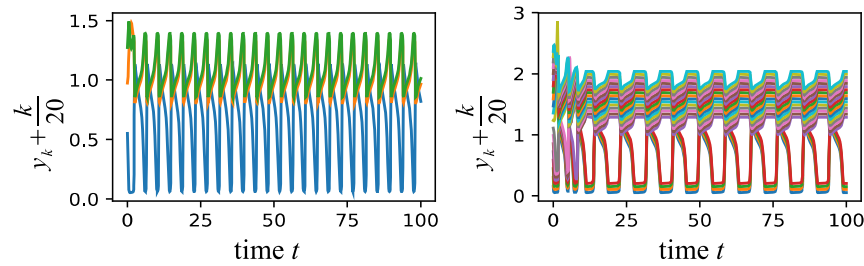

FIG. 4. $y_{k}$ time series obtained from numerically integrating Eqs. (30) and (31) for (a) $n=3$ and (b) $n=20$. Other parameters [in (a) and (b)]: $a=0.05, b=0.01, y_{\mathrm{tot}}=0.833 n$. Note that an offset of $k / 20$ was added to $y_{k}$ for better visualization of the individual time series in (a) and (b).

\section{EXAMPLE: CO ELECTRO-OXIDATION ON Pt}

As an example, we apply this result to a particular model, defined in Eqs. (30) and (31), that was derived from a more detailed model for $\mathrm{CO}$ electro-oxidation on Pt [11] (see Appendix C).

$$
\begin{aligned}
\frac{d}{d t} x_{k}(t)= & f\left(x_{k}(t), u(t)\right) \\
= & \frac{1-(1+a b) x_{k}(t)}{1+a-x_{k}(t)}-u(t)\left[1-x_{k}(t)\right] x_{k}(t), \\
y_{\text {tot }} & =\sum_{k=1}^{n} g\left(x_{k}(t), u(t)\right)=\sum_{k=1}^{n} y_{k}(t) \\
& =\sum_{k=1}^{n} u(t)\left[1-x_{k}(t)\right] x_{k}(t) .
\end{aligned}
$$

In short, $x_{k}$ is the CO coverage of electrode $k$ and $f\left(x_{k}(t), u(t)\right)$ comprises the rates of $\mathrm{CO}$ adsorption and desorption and the $u$-dependent reaction. Equation (31) determines the instantaneous change of the potential $u(t)$ such that the total current $y_{\text {tot }}$ is maintained constant despite changing $x_{k}$. Figures 3(c) and 3(d) exemplarily illustrate the three equilibria of Eqs. (30) and (31) for $n=3, a=0.05, b=0.01$ at $y_{\text {tot }}=0.833 n$. The two equilibria at $u=u_{1}$ and $u=u_{2}$ both have more than one component on the negative-slope branch, namely, three and two, respectively. As we have shown above, this means that these two equilibria are unstable because the clusters on the negative-slope branch have positive intracluster eigenvalues. Moreover, we can instantly rule out the possibility of oscillations at $u=u_{1}$, because all three components act inductively [Fig. 3(d), left]. In other words, the ensemble impedance lacks a capacitive component to form an $L C$ circuit. In contrast, the equilibrium at $u=u_{3}$ is intracluster stable for it has only one component in the negative-slope branch [Fig. 3(c)]. Moreover, at this equilibrium our necessary condition (29) for a Hopf bifurcation to occur is fulfilled [Fig. 3(e)]: While the change of CO coverage with $u$ always has a negative slope at the $x^{\text {eq }}$ values of the two clusters, the current depends nonmonotonically on the coverage and increases at the low $x^{\mathrm{eq}}$ values of the cluster on the active branch while it decreases at the higher $x^{\text {eq }}$ values of the electrode on the middle branch [Fig. 3(e)]. In fact, for the corresponding parameter values, sustained oscillations are observed [Fig. 4(a)]. 


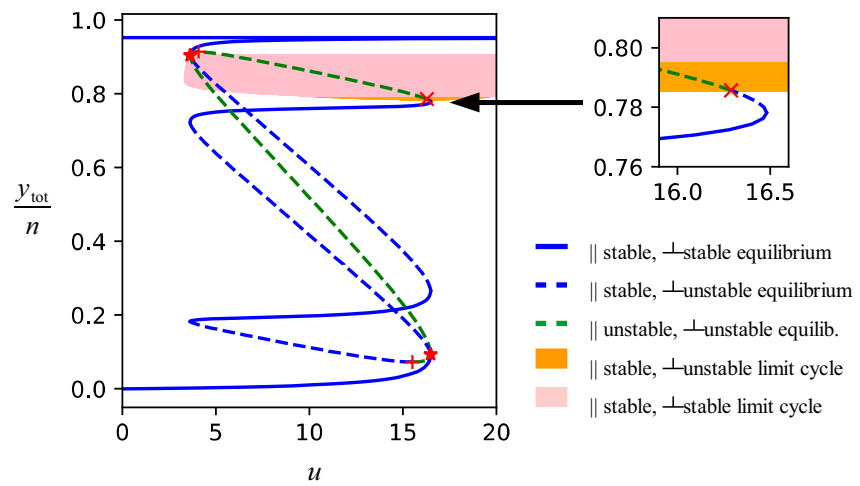

FIG. 5. Two-cluster limit sets of Eqs. (30) and (31) for $a=$ $0.05, b=0.01$, for a cluster size ratio of $4: 1$, valid for any $n$.

Our considerations so far revealed that Hopf bifurcations occur only in the intercluster subspace.

Hence a stable limit cycle can bifurcate only from an intracluster-stable equilibrium, just like the one in Fig. 4(a) bifurcated from the rightmost equilibrium in Fig. 3(c). As we saw, such an intracluster-stable equilibrium can have either no component on the middle, unstable branch of the $y$ - $u$ curve or one single component. For the former case, we were not able to come up with an example that has an oscillatory intercluster instability. For the latter case, the resulting limit cycle will not scale to larger ensemble sizes, because at some point the contribution of the one single component on the middle branch to the total current $y_{\text {tot }}$ becomes negligible, such that we are essentially back at the former case again. Thus, such a stable limit cycle will not persist for arbitrarily large $n$.

Yet, the model does support stable collective oscillations that do persist for arbitrarily large ensemble sizes. An example of this is given in Fig. 4(b), where we simulated 20 components and observed that they grouped in two clusters of size 16 and 4, oscillating in antiphase. The emergence of this collective oscillation is illustrated in the two-cluster bifurcation diagram shown in Fig. 5. For this, we numerically continued a four-component version of the system, in which each of the four components represented one of four clusters with sizes $n_{1}, n_{2}, n_{3}, n_{4}$ with $n_{1}+n_{2}+n_{3}+n_{4}=n$. We considered four clusters because this allowed us to divide each of the two clusters into two subclusters. In this way, we obtained the respective intracluster stability. To account for the cluster sizes, we modified Eq. (2) to $y_{\ell}=n_{\ell} g\left(x_{\ell}, u\right)$. We fixed $\left(n_{1}+n_{2}\right) / n=0.8,\left(n_{3}+n_{4}\right) / n=0.2$ to match the cluster size ratio we observed in the simulation, and $n_{2} / n=$
$0.16, n_{4} / n=0.04$. Note that the model depends only on the relative sizes of the clusters and not on the total ensemble size $n$. Besides the collective oscillation, Fig. 5 shows all the equilibria in which the ensemble is divided into two clusters in the same ratio as in Fig. 4(b), i.e., 4:1. Stable equilibria are shown as a solid line, unstable equilibria as a dashed line. Equilibria that are unstable within the cluster manifold and perpendicular to it are depicted in green, while those that are intercluster stable but intracluster unstable are shown as blue dashed lines.

As can be seen in the inset of Fig. 5, one of the transversally unstable branches undergoes a supercritical Hopf bifurcation, generating a limit cycle that is stable within the two-cluster manifold but unstable transversally to it (orange area). At somewhat larger values of $y_{\text {tot }}$, the limit cycle is stabilized in a type of transcritical bifurcation, generating stable collective oscillations (pink area).

\section{CONCLUSION}

In conclusion, we have presented a general mechanism of how an oscillation can emerge from an ensemble of globally coupled bistable, nonresonant components. Our considerations were motivated by two electrochemical systems, but our result applies to various physical situations.

Typically, for large ensembles the states of the individual components are not measurable. Rather, the state of the macroscopic system is characterized by some order parameter. Without the awareness that collective oscillations of the order parameter can be provoked by the mechanism discussed here, the natural interpretation would be that the oscillations emerge due to synchronization of individual oscillators. In view of our results, it might turn out that some observations of collective oscillations have to be reinterpreted.

\section{ACKNOWLEDGMENTS}

We thank A. Bonnefont and L. Bieler for fruitful discussions and M. Patzauer, F. Kemeth, and S. W. Haugland for critical reading of the manuscript. We also acknowledge financial support of TUM.solar in the framework of the Bavarian Collaborative Research Project Solar Technologies Go Hybrid (SolTech) and the DFG in the framework of the Cluster of Excellence "e-conversion." C.B. gratefully acknowledges the support of the Institute for Advanced Study at the Technical University of Munich through a Hans Fischer fellowship that made this work possible.

\section{APPENDIX A: EIGENVALUES AND EIGENVECTORS}

In the following we consider a more general scenario, in which the inner state $x(t)$ is a column vector of any arbitrary dimension $d$, so $x(t) \in \mathbb{R}^{d \times 1}, f_{x} \in \mathbb{R}^{d \times d}, f_{u} \in \mathbb{R}^{d \times 1}, g_{x} \in \mathbb{R}^{1 \times d}, g_{u} \in \mathbb{R}^{1 \times 1}$. Let $\mathbb{I}_{k} \in \mathbb{R}^{k \times k}$ be the identity matrix, let $\mathbf{0}^{j \times k}, \mathbf{1}^{j \times k} \in \mathbb{R}^{j \times k}$ be matrices of only zeros and only ones, respectively, and let $\otimes:\left(\mathbb{R}^{a \times b}, \mathbb{R}^{c \times d}\right) \rightarrow \mathbb{R}^{a c \times b d}$ denote the Kronecker product. Then, in the vicinity of a given ensemble equilibrium, we can rearrange the order of the entries in the ensemble state $\mathbf{x}(t)$ such that the individual bistable components are grouped by their respective component equilibria $x^{\mathrm{eq}, \ell}$. The linearization of the ensemble in the potentiostatic case takes the form

$$
\begin{aligned}
& \frac{d}{d t} \hat{\mathbf{x}}(t)=F_{x} \hat{\mathbf{x}}(t)+F_{u} \hat{u}, \\
& \hat{y}_{\mathrm{tot}}(t)=G_{x} \hat{\mathbf{x}}(t)+G_{u} \hat{u},
\end{aligned}
$$


and in the galvanostatic case

$$
\begin{gathered}
\frac{d}{d t} \hat{\mathbf{x}}(t)=\overbrace{\left(F_{x}-F_{u} G_{u}^{-1} G_{x}\right)}^{=: \tilde{F}_{x}} \hat{\mathbf{x}}(t)+F_{u} G_{u}^{-1} \hat{y}_{\mathrm{tot}}, \\
\hat{u}(t)=-G_{u}^{-1} G_{x} \hat{\mathbf{x}}(t)+G_{u}^{-1} \hat{y}_{\mathrm{tot}} .
\end{gathered}
$$

Here,

$$
\begin{aligned}
& \hat{\mathbf{x}}(t):=\left(\begin{array}{c}
x_{1}(t)-x^{\mathrm{eq}, 1} \\
x_{2}(t)-x^{\mathrm{eq}, 1} \\
\vdots \\
x_{n_{1}}(t)-x^{\mathrm{eq}, 1} \\
x_{n_{1}+1}(t)-x^{\mathrm{eq}, 2} \\
x_{n_{1}+2}(t)-x^{\mathrm{eq}, 2} \\
\vdots \\
x_{n_{1}+n_{2}}(t)-x^{\mathrm{eq}, 2} \\
x_{n_{1}+n_{2}+1}(t)-x^{\mathrm{eq}, 3} \\
x_{n_{1}+n_{2}+2}(t)-x^{\mathrm{eq}, 3} \\
\vdots \\
x_{n_{1}+n_{2}+n_{3}}(t)-x^{\mathrm{eq}, 3}
\end{array}\right) \\
& F_{x}:=\left(\begin{array}{lll}
\mathbb{I}_{n_{1}} \otimes f_{x}\left(x^{\mathrm{eq}, 1}\right) & & \\
& \mathbb{I}_{n_{2}} \otimes f_{x}\left(x^{\mathrm{eq}, 2}\right) & \\
& & \mathbb{I}_{n_{3}} \otimes f_{x}\left(x^{\mathrm{eq}, 3}\right)
\end{array}\right) \quad \in \mathbb{R}^{n d \times n d,}, \\
& F_{u}:=\left(\begin{array}{l}
\mathbf{1}^{n_{1} \times 1} \otimes f_{u}\left(x^{\mathrm{eq}, 1}\right) \\
\mathbf{1}^{n_{2} \times 1} \otimes f_{u}\left(x^{\mathrm{eq}, 2}\right) \\
\mathbf{1}^{n_{3} \times 1} \otimes f_{u}\left(x^{\mathrm{eq}, 3}\right)
\end{array}\right) \quad \in \mathbb{R}^{n d \times 1},
\end{aligned}
$$

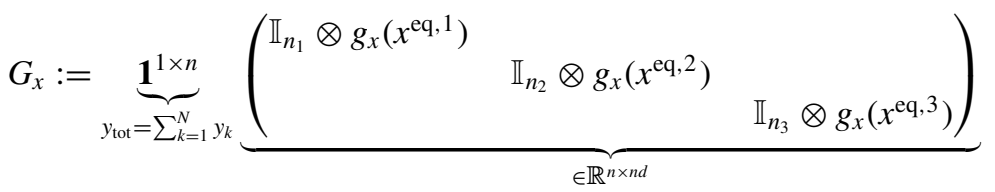

$$
\begin{aligned}
& =\left(\mathbf{1}^{1 \times n_{1}} \otimes g_{x}\left(x^{\mathrm{eq}, 1}\right), \quad \mathbf{1}^{1 \times n_{2}} \otimes g_{x}\left(x^{\mathrm{eq}, 2}\right), \quad \mathbf{1}^{1 \times n_{3}} \otimes g_{x}\left(x^{\mathrm{eq}, 3}\right)\right) \quad \in \mathbb{R}^{1 \times n d}, \\
& G_{u}:=\underbrace{\mathbf{1}^{1 \times n}}_{y_{\mathrm{tot}}=\sum_{k=1}^{n} y_{k}}\left(\begin{array}{l}
\mathbf{1}^{n_{1} \times 1} \otimes g_{u}\left(x^{\mathrm{eq}, 1}\right) \\
\mathbf{1}^{n_{2} \times 1} \otimes g_{u}\left(x^{\mathrm{eq}, 2}\right) \\
\mathbf{1}^{n_{3} \times 1} \otimes g_{u}\left(x^{\mathrm{eq}, 3}\right)
\end{array}\right) \\
& =n_{1} g_{u}\left(x^{\mathrm{eq}, 1}\right)+n_{2} g_{u}\left(x^{\mathrm{eq}, 2}\right)+n_{3} g_{u}\left(x^{\mathrm{eq}, 3}\right) \quad \in \mathbb{R}^{1 \times 1} .
\end{aligned}
$$

\section{Intracluster eigenvalues and eigenvectors}

If $x(t)$ is a $d$-dimensional vector, we obtain the following intra cluster eigenvalues $\lambda_{\ell}^{\perp}$ and intracluster eigenvectors $\mathbf{v}_{\ell}^{\perp}$ of the ensemble Jacobian $\tilde{F}_{x}$ under current control:

$$
\begin{gathered}
\left(\tilde{F}_{x}-\lambda_{\ell}^{\perp} \mathbb{I}_{n d}\right) \mathbf{v}_{\ell}^{\perp}=0, \\
\mathbf{v}_{1}^{\perp}=\left(\begin{array}{c}
\mathbf{w}_{1}^{\perp} \otimes v_{1}^{\perp} \\
\mathbf{0}^{n_{2} d \times 1} \\
\mathbf{0}^{n_{3} d \times 1}
\end{array}\right), \\
\mathbf{v}_{2}^{\perp}=\left(\begin{array}{c}
\mathbf{0}^{n_{1} d \times 1} \\
\mathbf{w}_{2}^{\perp} \otimes v_{2}^{\perp} \\
\mathbf{0}^{n_{3} d \times 1}
\end{array}\right),
\end{gathered}
$$




$$
\mathbf{v}_{3}^{\perp}=\left(\begin{array}{c}
\mathbf{0}^{n_{1} d \times 1} \\
\mathbf{0}^{n_{2} d \times 1} \\
\mathbf{w}_{3}^{\perp} \otimes v_{3}^{\perp}
\end{array}\right)
$$

in which

$$
\begin{array}{clll}
\mathbf{w}_{\ell}^{\perp} & \in \mathbb{R}^{n_{\ell} \times 1} & : \quad \text { entries sum up to zero, i.e., } \mathbf{1}^{1 \times n_{\ell}} \mathbf{w}_{\ell}^{\perp}=0, \\
v_{\ell} & \in \mathbb{R}^{d \times 1} & : \quad \text { an eigenvector of } f_{x}\left(x^{\mathrm{eq}, \ell}\right), \\
\lambda_{\ell}^{\perp} & \in \mathbb{R}^{\mathrm{R}} & : \quad \text { the eigenvalue of } f_{x}\left(x^{\mathrm{eq}, \ell}\right) \text { to the eigenvector } v_{\ell}^{\perp} .
\end{array}
$$

There are $\sum_{\ell=1}^{3} d \max \left(n_{\ell}-1,0\right)$ such intracluster eigenvectors $\mathbf{v}_{\ell}^{\perp}$ in total, since we have $\max \left(n_{\ell}-1,0\right)$ dimensions to choose $\mathbf{w}_{\ell}^{\perp}$ from and $d$ dimensions for $v_{\ell}^{\perp}$. The eigenvalues $\lambda_{\ell}^{\perp}$ are $\max \left(n_{\ell}-1,0\right)$-fold degenerate for each $\ell$, respectively.

Checking Eq. (A6) for $\ell=1$, exemplarily,

$$
\begin{aligned}
& \left(\tilde{F}_{x}-\lambda_{1}^{\perp} \mathbb{I}_{n d}\right) \mathbf{v}_{1}^{\perp} \stackrel{(16)}{=}\left(F_{x}-F_{u} G_{u}^{-1} G_{x}-\lambda_{1}^{\perp} \mathbb{I}_{n d}\right) \mathbf{v}_{1}^{\perp} \\
& =F_{x} \mathbf{v}_{1}^{\perp}-F_{u} G_{u}^{-1} G_{x} \mathbf{v}_{1}^{\perp}-\lambda_{1}^{\perp} \mathbb{I}_{n d} \mathbf{v}_{1}^{\perp} \\
& =\left(\begin{array}{c}
{\left[\mathbb{I}_{n_{1}} \otimes f_{x}\left(x^{\mathrm{eq}, 1}\right)\right]\left[\mathbf{w}_{1} \otimes v_{1}^{\perp}\right]} \\
\mathbf{0}^{\left(n_{2}+n_{3}\right) d \times 1}
\end{array}\right)-F_{u} G_{u}^{-1}\left(\begin{array}{c}
{\left[\mathbf{1}^{1 \times n_{1}} \otimes g_{x}\left(x^{\mathrm{eq}, 1}\right)\right]\left[\mathbf{w}_{1} \otimes v_{1}^{\perp}\right]} \\
\mathbf{0}^{\left(n_{2}+n_{3}\right) d \times 1}
\end{array}\right)-\lambda_{1}^{\perp} \mathbf{v}_{1}^{\perp} \\
& =\left(\begin{array}{c}
{\left[\mathbb{I}_{n_{1}} \mathbf{w}_{1}^{\perp}\right] \otimes\left[f_{x}\left(x^{\mathrm{eq}, 1}\right) v_{1}^{\perp}\right]} \\
\mathbf{0}^{\left(n_{2}+n_{3}\right) d \times 1}
\end{array}\right)-F_{u} G_{u}^{-1} \underbrace{\left(\begin{array}{c}
\mathbf{1}^{1 \times n_{1}} \mathbf{w}^{\left(n_{1}+n_{2}+n_{3}\right) d \times 1} \\
\left.\mathbf{w}_{1}^{\perp}\right] \otimes\left[g_{x}\left(x^{\mathrm{eq}, 1}\right) v_{1}^{\perp}\right]
\end{array}\right)}_{\substack{\text { def of } \mathbf{w}_{1}^{\perp} \\
=}}-\lambda_{1}^{\perp} \mathbf{v}_{1}^{\perp} \\
& =\left(\begin{array}{c}
\mathbf{w}_{1}^{\perp} \otimes \lambda_{1}^{\perp} v_{1}^{\perp} \\
\mathbf{0}^{\left(n_{2}+n_{3}\right) d \times 1}
\end{array}\right)-\lambda_{1}^{\perp} \mathbf{v}_{1}^{\perp} \\
& =\lambda_{1}^{\perp} \mathbf{v}_{1}^{\perp}-\lambda_{1}^{\perp} \mathbf{v}_{1}^{\perp} \\
& =0 \text {. }
\end{aligned}
$$

The cases $\ell=2$ and $\ell=3$ can be shown analogously.

\section{Intercluster eigenvalues and eigenvectors}

Previously, we described intracluster eigenvectors $\mathbf{v}_{\ell}^{\perp}$ corresponding to perturbations within single clusters. In contrast, the following intercluster eigenvectors $\mathbf{v}^{\|}$each perturb more than one cluster but leave the components within each cluster synchronous to each other. We claim that the following equation (A17) describes the remaining $3 d$ (or less if a cluster is empty) eigenvectors $\mathbf{v}^{\|}$of the current-control Jacobian $\tilde{F}_{x}$ corresponding to the eigenvalues $\lambda^{\|}$:

$$
\left(\tilde{F}_{x}-\lambda^{\|} \mathbb{I}_{n d}\right) \underbrace{\left(F_{x}-\lambda^{\|} \mathbb{I}_{n d}\right)^{-1} F_{u}}_{=: \mathbf{v} \|}=0 .
$$

However, such an eigenvector $\mathbf{v}^{\|}$only exists if $\left(F_{x}-\lambda^{\|} \mathbb{I}_{n d}\right)$ is invertible. $\left(F_{x}-\lambda^{\|} \mathbb{I}_{n d}\right)$ is invertible if and only if we assume $\lambda^{\|} \neq \lambda_{\ell}^{\perp}$ for all $\lambda_{\ell}^{\perp}$. This is because if $\lambda^{\|} \neq \lambda_{\ell}^{\perp}$, then and only then $\lambda^{\|}$is not an eigenvalue of $F_{x}$, which are $n_{\ell} \times \lambda_{\ell}^{\perp}$, for $\ell=1,2,3$ [see the definition of $F_{x}$ in Eq. (A2)]. However, if $\lambda^{\|}$is not an eigenvalue of $F_{x}$, then and only then $\operatorname{det}\left(F_{x}-\lambda^{\|} \mathbb{I}_{n d}\right) \neq 0$, i.e., $\left(F_{x}-\lambda^{\|} \mathbb{I}_{n d}\right)$ is invertible.

We further claim that the corresponding eigenvalues $\lambda^{\|}$are obtained by solving Eq. (A18), which might be difficult in general:

$$
\underbrace{-G_{x} \underbrace{=}_{(3),(4)} \frac{\mathcal{L}\left(\hat{y}_{\mathrm{tot}}\right\}(\lambda \|)}{\mathcal{L}\{\hat{u}\}(\lambda \|)}=: Y_{\mathrm{tot}}(\lambda \|)}_{\equiv \mathbf{v} \|}=0 .
$$

$\mathcal{L}$ denotes the Laplace transformation, and in Eq. (A18) we used $\mathcal{L}\left\{\frac{d}{d t} \hat{\mathbf{x}}\right\}\left[\lambda^{\|}\right]=\lambda^{\|} \mathcal{L}\{\hat{\mathbf{x}}\}\left[\lambda^{\|}\right]$. Equations (A17) and (A18) say that, at the eigenvalues $\lambda^{\|}$of $\tilde{F}_{x}$, the total admittance $Y_{\text {tot }}\left(\lambda^{\|}\right)$vanishes. Notice that $Y_{\text {tot }}\left(\lambda^{\|}\right)$is only defined if $\lambda^{\|}$is not an eigenvalue of $F_{x}$. Analogously, the total impedance $Z_{\text {tot }}\left(\lambda^{\|}\right):=Y_{\text {tot }}^{-1}\left(\lambda^{\|}\right)$is only defined if $\lambda^{\|}$is not an eigenvalue of $\tilde{F}_{x}$. Let us confirm Eq. (A17):

$$
\begin{aligned}
\left(\tilde{F}_{x}-\lambda^{\|} \mathbb{I}_{n d}\right) \mathbf{v}^{\|} \stackrel{\stackrel{(16)}{=}}{=}\left(F_{x}-F_{u} G_{u}^{-1} G_{x}-\lambda^{\|} \mathbb{I}_{n d}\right) \mathbf{v}^{\|} \\
=\left(F_{x}-\lambda^{\|} \mathbb{I}_{n d}\right) \mathbf{v}^{\|}-F_{u} G_{u}^{-1} \underbrace{G_{x} \mathbf{v}^{\|}}_{\stackrel{(A 18)}{=} G_{u}}
\end{aligned}
$$




$$
\begin{aligned}
& \stackrel{\left(A_{117)}\right.}{=}\left(F_{x}-\lambda^{\|} \mathbb{I}_{n d}\right)\left(F_{x}-\lambda^{\|} \mathbb{I}_{n d}\right)^{-1} F_{u}-F_{u} G_{u}^{-1} G_{u} \\
& =F_{u}-F_{u} \\
& =0 .
\end{aligned}
$$

\section{APPENDIX B: CIRCUIT REPRESENTATION}

The component equation

$$
\begin{aligned}
\frac{d}{d t} x_{k}(t) & =f\left(x_{k}(t), u(t)\right), \\
y_{k}(t) & =g\left(x_{k}(t), u(t)\right)
\end{aligned}
$$

can be linearized

$$
\begin{gathered}
\frac{d}{d t} \hat{x}(t)=f_{x} \hat{x}(t)+f_{u} \hat{u}(t), \\
\hat{y}_{k}(t)=g_{x} \hat{x}(t)+g_{u} \hat{u}(t)
\end{gathered}
$$

and Laplace transformed

$$
\begin{gathered}
i \omega \mathcal{L}\{\hat{x}\}(i \omega)=f_{x} \mathcal{L}\{\hat{x}\}(i \omega)+f_{u} \mathcal{L}\{\hat{u}(t)\}(i \omega), \\
\mathcal{L}\left\{\hat{y}_{k}\right\}(i \omega)=g_{x} \mathcal{L}\{\hat{x}\}(i \omega)+g_{u} \mathcal{L}\{\hat{u}(t)\}(i \omega) .
\end{gathered}
$$

Eliminating $\mathcal{L}\{\hat{x}\}(i \omega)$, we get the admittance $Y_{k}(i \omega)$ :

$$
\mathcal{L}\left\{\hat{y}_{k}\right\}(i \omega)=\underbrace{\left[g_{x}\left(i \omega-f_{x}\right)^{-1} f_{u}+g_{u}\right]}_{Y_{k}(i \omega)} \mathcal{L}\{\hat{u}(t)\}(i \omega) .
$$

In comparison, the circuit in Fig. 3(a) yields the same, identifying $R_{1}, R_{2}, L$ with the respective values of the three electrical components.

$$
\begin{aligned}
Y_{k}(i \omega) & =\left(i \omega L+R_{2}\right)^{-1}+R_{1}^{-1} \\
& =\left[i \omega\left(g_{x} f_{u}\right)^{-1}-f_{x}\left(g_{x} f_{u}\right)^{-1}\right]^{-1}+g_{u} \\
& =g_{x}\left[i \omega-f_{x}\right]^{-1} f_{u}+g_{u} .
\end{aligned}
$$

Analogously, the same is true for the circuit in Fig. 3(b), with $R_{1}, R_{2}, C$.

$$
\begin{aligned}
Y_{k}(i \omega) & =\left[(i \omega C)^{-1}+R_{2}\right]^{-1}+R_{1}^{-1} \\
& =\left[-(i \omega)^{-1} f_{x}^{2}\left(g_{x} f_{u}\right)^{-1}+f_{x}\left(g_{x} f_{u}\right)^{-1}\right]^{-1}+\left(g_{u}-f_{x}^{-1} g_{x} f_{u}\right) \\
& =g_{x}\left[\frac{1}{-(i \omega)^{-1} f_{x}^{2}+f_{x}}-\frac{1}{f_{x}}\right] f_{u}+g_{u} \\
& =g_{x}\left[\frac{-i \omega}{f_{x}^{2}-i \omega f_{x}}-\frac{f_{x}-i \omega}{f_{x}^{2}-i \omega f_{x}}\right] f_{u}+g_{u} \\
& =g_{x}\left[\frac{-f_{x}}{f_{x}^{2}-i \omega f_{x}}\right] f_{u}+g_{u} \\
& =g_{x}\left[i \omega-f_{x}\right]^{-1} f_{u}+g_{u} .
\end{aligned}
$$

\section{APPENDIX C: DERIVATION OF THE MODEL FOR CO ELECTRO-OXIDATION ON Pt}

We derived our model from the following, more detailed model for the $\mathrm{CO}$ adsorption on a platinum microelectrode, which is described in detail in Ref. [11].

$$
\begin{gathered}
\frac{d}{d t} C_{i}=\frac{2 D}{\delta^{2}}\left(C_{\mathrm{b}}-C_{i}\right)-\frac{S_{\mathrm{tot}}}{\delta}\left[k_{\mathrm{ads}} C_{i}\left(1-\theta_{i}\right)-k_{\mathrm{des}} \theta_{i}\right], \\
\frac{d}{d t} \theta_{i}=k_{\mathrm{ads}} C_{i}\left(1-\theta_{i}\right)-k_{\mathrm{des}} \theta_{i}-k_{\mathrm{reac}} \theta_{i}\left(1-\theta_{i}\right) \exp \left(\alpha f \phi_{i}\right), \\
J_{i}=2 F S_{\mathrm{tot}} k_{\mathrm{reac}} \theta_{i}\left(1-\theta_{i}\right) \exp \left(\alpha f \phi_{i}\right),
\end{gathered}
$$


Our simplified model captures the steady states of Eqs. (C1) and (C2). Its impedance is not exactly the same as in the original model, but it still provides oscillations as an ensemble.

To obtain our simplified version, we set $d C_{i} / d t$ to zero to eliminate $C_{i}$, so Eq. (C2) becomes Eq. (C4).

$$
\begin{gathered}
\frac{d}{d t} \theta_{i}=\frac{D C_{\mathrm{b}} k_{\mathrm{ads}}-\left(D C_{\mathrm{b}} k_{\mathrm{ads}}+D k_{\mathrm{des}}\right) \theta_{i}}{D+k_{\mathrm{ads}} \delta S_{\mathrm{tot}}-k_{\mathrm{ads}} \delta S_{\mathrm{tot}} \theta_{i}}-k_{\mathrm{reac}} \theta_{i}\left(1-\theta_{i}\right) \exp \left(\alpha f \phi_{i}\right), \\
J_{i}=2 F S_{\mathrm{tot}} k_{\mathrm{reac}} \theta_{i}\left(1-\theta_{i}\right) \exp \left(\alpha f \phi_{i}\right) .
\end{gathered}
$$

Then, defining

$$
\begin{gathered}
\tau:=\frac{D C_{\mathrm{b}}}{\delta S_{\mathrm{tot}}} t, \\
u_{i}:=\frac{\delta S_{\mathrm{tot}}}{D C_{\mathrm{b}}} k_{\mathrm{reac}} \exp \left(\alpha f \phi_{i}\right), \\
y_{i}:=J_{i} \frac{\delta}{2 F D C_{\mathrm{b}}}, \\
a:=\frac{D}{k_{\mathrm{ads}} \delta S_{\mathrm{tot}}}, \\
b:=\frac{k_{\mathrm{des}} \delta S_{\mathrm{tot}}}{D C_{\mathrm{b}}},
\end{gathered}
$$

we are left with

$$
\begin{gathered}
\frac{d}{d \tau} \theta_{i}=\frac{1-(1+a b) \theta_{i}}{1+a-\theta_{i}}-\theta_{i}\left(1-\theta_{i}\right) u_{i}, \\
y_{i}=\theta_{i}\left(1-\theta_{i}\right) u_{i},
\end{gathered}
$$

in which $a$ and $b$ are small, positive constants. We rename $\tau$ to $t$ and $\theta_{i}$ to $x_{i}$ and change the values of $a$ and $b$ to make the system less stiff, but we keep them much smaller than 1 .

[1] N. E. Kouvaris, H. Kori, and A. S. Mikhailov, Traveling and pinned fronts in bistable reaction-diffusion systems on networks, PLoS One 7, e45029 (2012).

[2] N. E. Kouvaris and A. S. Mikhailov, Feedback-induced stationary localized patterns in networks of diffusively coupled bistable elements, EPL 102, 16003 (2013).

[3] N. E. Kouvaris, M. Sebek, A. S. Mikhailov, and I. Z. Kiss, Selforganized stationary patterns in networks of bistable chemical reactions, Angew. Chem. 128, 13461 (2016).

[4] D. A. Crespo-Yapur, A. Bonnefont, R. Schuster, K. Krischer, and E. R. Savinova, Sequential activation and oscillations of globally coupled microelectrodes during a bistable reaction, ChemElectroChem 1, 1046 (2014).

[5] D. A. Crespo-Yapur, A. Bonnefont, R. Schuster, K. Krischer, and E. R. Savinova, Cooperative behaviour of Pt microelectrodes during CO bulk electrooxidation, ChemPhysChem 14, 1117 (2013).

[6] W. Dreyer, J. Jamnik, C. Guhlke, R. Huth, J. Moškon, and M. Gaberšček, The thermodynamic origin of hysteresis in insertion batteries, Nat. Mater. 9, 448 (2010).

[7] W. Dreyer, C. Guhlke, and R. Huth, The behavior of a manyparticle electrode in a lithium-ion battery, Phys. D (Amsterdam, Neth.) 240, 1008 (2011).

[8] C. Delmas, M. Maccario, L. Croguennec, F. Le Cras, and F. Weill, Lithium deintercalation in $\mathrm{LiFePO}_{4}$ nanoparticles via a domino-cascade model, Nat. Mater. 7, 665 (2008).
[9] G. Brunetti, D. Robert, P. Bayle-Guillemaud, J. L. Rouvière, E. F. Rauch, J. F. Martin, J. F. Colin, F. Bertin, and C. Cayron, Confirmation of the domino-cascade model by $\mathrm{LiFePO}_{4} / \mathrm{FePO}_{4}$ precession electron diffraction, Chem. Mater. 23, 4515 (2011).

[10] Y. Li, F. El Gabaly, T. R. Ferguson, R. B. Smith, N. C. Bartelt, J. D. Sugar, K. R. Fenton, D. A. Cogswell, A. L. D. Kilcoyne, T. Tyliszczak, M. Z. Bazant, and W. C. Chueh, Current-induced transition from particle-by-particle to concurrent intercalation in phase-separating battery electrodes, Nat. Mater. 13, 1149 (2014).

[11] S. Bozdech, Y. Biecher, E. R. Savinova, R. Schuster, K. Krischer, and A. Bonnefont, Oscillations in an array of bistable microelectrodes coupled through a globally conserved quantity, Chaos 28, 045113 (2018).

[12] M. T. M. Koper, T. J. Schmidt, N. M. Marković, and P. N. Ross, Potential oscillations and S-shaped polarization curve in the continuous electro-oxidation of $\mathrm{CO}$ on platinum single-crystal electrodes, J. Phys. Chem. B 105, 8381 (2001).

[13] R. Morschl, J. Bolten, A. Bonnefont, and K. Krischer, Pattern formation during $\mathrm{CO}$ electrooxidation on thin $\mathrm{Pt}$ films studied with spatially resolved infrared absorption spectroscopy, J. Phys. Chem. C 112, 9548 (2008).

[14] S. Bozdech, K. Krischer, D. A. Crespo-Yapur, E. Savinova, and A. Bonnefont, $1 / f^{2}$ noise in bistable electrocatalytic reactions on mesoscale electrodes, Faraday Discuss. 193, 187 (2016).

[15] R. Imbihl and G. Ertl, Oscillatory kinetics in heterogeneous catalysis, Chem. Rev. (Washington, DC, U. S.) 95, 697 (1995). 
[16] Control System Fundamentals, The Control Handbook, edited by W. S. Levine (CRC, Boca Raton, 2010).

[17] W. L. Ku, M. Girvan, and E. Ott, Dynamical transitions in large systems of mean field-coupled Landau-Stuart oscillators: Extensive chaos and cluster states, Chaos 25, 123122 (2015).
[18] T. R. Ferguson and M. Z. Bazant, Nonequilibrium thermodynamics of porous electrodes, J. Electrochem. Soc. 159, A1967 (2012).

[19] Y. A. Kuznetsov, Andronov-Hopf bifurcation, Scholarpedia 1, 1858 (2006) Revision No. 90964. 